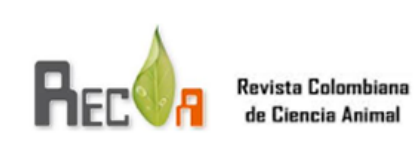

Revista Colombiana de Ciencia Animal

ISSN: 2027-4297

reciaeditor@unisucre.edu

Universidad de Sucre

Colombia

\title{
Estimación de la calidad de la dieta en sistemas silvopastoriles mediante la cuantificación del nitrógeno fecal
}

Portilla-Pinzón, Danilo; Barragán-Hernández, Wilson; Carvajal-Bazurto, Christian; Cajas-Girón, Yasmin Estimación de la calidad de la dieta en sistemas silvopastoriles mediante la cuantificación del nitrógeno fecal Revista Colombiana de Ciencia Animal, vol. 11, núm. 1, 2019

Universidad de Sucre, Colombia

DOI: https://doi.org/10.24188/recia.v0.n0.2019.691

Los autores permiten a RECIA reimprimir el material publicado en él. En caso de que un autor quiera traducir o usar una publicación parcial o completa de nuestro Diario, el autor debe obtener un permiso por escrito del editor de la revista.

Esta obra está bajo una Licencia Creative Commons Atribución 4.0 Internacional. 


\section{Estimación de la calidad de la dieta en sistemas silvopastoriles mediante la cuantificación del nitrógeno fecal}

Estimation of the quality of the diet in silvopastoral systems through the quantification of fecal nitrogen

Danilo Portilla-Pinzón

DOI: https://doi.org/10.24188/recia.v0.n0.2019.691

Corporación Colombiana de Investigación Agropecuaria

(Agrosavia), Centro de Investigación El Nus. San Roque,

Colombia., Colombia

dportilla@agrosavia.co

(iD http://orcid.org/0000-0001-6125-036X

Recepción: 29 Abril 2018

Aprobación: 27 Diciembre 2018

Wilson Barragán-Hernández

Corporación Colombiana de Investigación Agropecuaria

(Agrosavia), Centro de Investigación Turipaná. Cereté,

Colombia, Colombia

wbarraganh@agrosavia.co

(D) http://orcid.org/0000-0003-3528-4296

Christian Carvajal-Bazurto

Corporación Colombiana de Investigación Agropecuaria

(Agrosavia), Centro de Investigación Nataima. Ibagué,

Colombia, Colombia

ccarvajal@cagrosavia.co

http://orcid.org/0000-0001-8668-5562

Yasmin Cajas-Girón

Corporación Colombiana de Investigación Agropecuaria

(Agrosavia). Colombia, Colombia

yasminscg@gmail.com

(iD http://orcid.org/0000-0002-8740-9567

\section{Resumen:}

Con el objeto de evaluar la calidad de la dieta consumida por bovinos en sistemas silvopastoriles (SSP), mediante su estimación a través del nitrógeno fecal (NF), se compararon cuatro arreglos que variaron en su composición vegetal, con especies arbustivas (L. leucocephala y C. cujete), arbóreas (A. saman, G. ulmifolia y C. grandis) y maderables (P. quinata y S. macrophylla), contra un tratamiento sin árboles (M. maximum). A cada tratamiento fueron asignadas 4 vacas Romosinuano $\mathrm{x}$ Holstein $\mathrm{x}$ Cebú (436 $\pm 64,2$ $\mathrm{kg}$ ) a las cuales se les colectó heces vía rectal. Las muestras de heces fueron secadas y sometidas a análisis de nitrógeno (total, soluble e insoluble en FDN), FDN y materia orgánica (MO). Con el NF se estimó la cantidad de proteína fecal (PCF), proteína cruda $(\mathrm{PC})$ y la digestibilidad de la dieta consumida en cada SSP. Se observó efecto significativo $(\mathrm{p}<0.01)$ del tratamiento sobre la cantidad de NF, presentado como PCF. El tratamiento Pasto-Arbust-Arbor-Mad obtuvo un valor de 132.19 g.kg MO-1, con diferencia significativa de los tratamientos Pasto, Pasto-Arbus y Pasto-Arbor, con registros de 119.77, 116.77 y 118.91 g.kg MO-1 respectivamente. No se registró efecto del tratamiento en las fracciones de MO, FDN y el contenido de nitrógeno soluble e insoluble en FDN. La digestibilidad y la PC de la dieta consumida estimada a través del NF varió de 61.45\% a 65.24\% y de 10.28\% a $12.02 \%$ respectivamente. La cuantificación del NF permitió revelar la calidad de la dieta que consumen los bovinos en ambientes SSP de estructura vegetal compleja.

Palabras ClaVe: Árboles, arbustos, calidad de forraje, materia orgánica fecal, proteína .

\section{NotAS DE AUTOR}




\begin{abstract}
:
In order to evaluate the quality of the diet consumed by bovines in multiestrata silvopastoral systems (SSP) through estimation fecal nitrogen's (NF), were compared four silvopastoral arrangements with variation in their vegetal composition versus a treeless prairie (M. maximum). Each arrangement was composed by shrubs (L. leucocephala y C. cujete), trees (A. saman, G. ulmifolia y C. grandis) and timbers species (P. quinata y S. macrophylla). Four cow Romosinuano x Holstein x Cebu (436 $\pm 64,2 \mathrm{~kg})$ were assigned in each treatment. In each cow feces were collected directly from the rectum. The samples were dried and analyzed for nitrogen (total, soluble and insoluble in NDF), NDF and the organic matter. The information obtained in NF was used for estimated the fecal crude protein (FCP), crude protein (CP) and digestibility in intake of each SSP. The NF presented as FCP was affected by treatment $(\mathrm{p}<0.1)$. The treatment Pasto-Arbust-Arbor-Mad showed the higher value (132.19 g.kg MO-1) significantly different from treatment Pasto, Pasto-Arbust y Pasto-Arbor, with 119.77, 116.77 y 118.91 g.kg MO-1 respectively. MO, NDF and the soluble and insoluble nitrogen no showed statistical difference between treatments. The intake digestibility and CP estimated through NF ranged from $61.45 \%$ to $65.24 \%$ and from 10.28 to 12.02 respectively. NF quantification allowed to show the quality of intake in the complex plant structure of the silvopastoral systems.
\end{abstract}

KEYWORDS: Fecal organic matter, forage quality, protein, tree, shurbs .

\title{
INTRODUCCIÓN
}

El nivel de producción y la calidad de los productos que se puede obtener de bovinos que forrajean áreas con mezclas complejas de vegetación, está relacionada a su habilidad para seleccionar una dieta apropiada que cubra sus requerimientos nutricionales $(1,2)$. Esta habilidad, depende de las decisiones de forrajeo y es directamente proporcional al consumo de materia seca, la concentraciones de nutrientes y la digestibilidad de la dieta seleccionada (3), factores que junto a los compuestos secundarios, se consideran determinantes de la calidad de un forraje (4).

Bajo sistemas con una composición vegetal diversa y suficiente para que los animales tengan la capacidad de selección, tal como se considera en sistemas silvopastoriles, la determinación de la calidad de la dieta se vuelve más difícil de estimar debido al carácter complejo de dicha selección y a las limitaciones técnicas para considerar la diversidad vegetal, lo que dificulta el establecimiento relaciones con las respuestas productivas, e incluso comparar sistemas de producción con diversidad vegetal, contra esquemas de producción en monocultivo $(2,5)$.

Es así que la comprensión de las decisiones de forrajeo a través del valor nutritivo de la dieta seleccionada por los rumiantes son los principales retos que enfrentan los científicos y productores, debido a que los métodos tradicionales para determinar la calidad nutritiva bajo forrajeo libre tienen grandes limitaciones, las cuales se agudizan al considerar una oferta forrajera diversa, siendo el reto más desafiante el muestreo representativo de la dieta ingerida en las áreas con múltiples especies (6).

Los estudios sobre la selección de la dieta consumida por rumiantes bajos sistemas de pastoreo y/o ramoneo requieren con frecuencia el uso de aproximaciones indirectas y simples para determinar la calidad del alimento ingerido (7). Al respecto se ha considerado que las tendencias en la calidad nutritiva fecal pueden estar asociadas en varios niveles con las tendencias en la calidad de la dieta y desempeño animal (8), por lo que un gran número de trabajos y debates se han orientado desde comienzos de los años 40 para establecer relaciones entre índices fecales y la calidad de la dieta seleccionada. La mayor parte de estos, llevados a cabo en el estudio de interacciones ecológicas de herbívoros silvestres bajo diversos nichos o en rumiantes domésticos bajo sistemas extensivos en pastizales (9).

Entre los índices fecales más usados, la concentración de nitrógeno (NF) y sus fracciones soluble e insoluble han sido los más estudiados debido a las altas correlaciones que presentan con la digestibilidad, contenido de proteína y un menor grado con el consumo de materia orgánica y contenidos de taninos (10). Por tal motivo, durante las últimas décadas se han venido desarrollando modelos empíricos, basados en ecuaciones de regresión lineal y no lineal, para la predicción de la calidad de la dieta seleccionada por rumiantes en forrajeo libre a partir de la concentración de NF $(7,11,12)$. Sin embargo, la naturaleza empírica de estos modelos 
hace que sus aplicaciones solo sean válidas bajo condiciones muy similares a las que se han desarrollado (13), aunque permiten evidenciar el principio de las relaciones que existen entre las variables. El conocimiento de estas relaciones permite inferir de forma general el estado nutricional de los animales y formular hipótesis al comparar diferentes condiciones de forrajeo, las cuales se pueden apoyar con la respuesta productiva de los animales y estudios más específicos.

El objetivo de este estudio fue determinar el efecto de los sistemas silvopastoriles en la concentración de nitrógeno en la materia orgánica fecal y su relación con la estimación de la calidad de la dieta consumida.

\section{MATERIALES Y MÉTODOS}

Área de estudio y diseño experimental: La evaluación se llevó a cabo en el centro de investigación Turipana\#, adscrito a la Corporación colombiana de investigación agropecuaria (AGROSAVIA), localizado en Cerete\#, Córdoba, Colombia ( $8^{\circ} 51^{\prime} \mathrm{N}, 75^{\circ} 49^{\prime} \mathrm{W}$, altitud de $18 \mathrm{msnm}$ ). La zona presenta dos periodos climáticos definidos, un periodo de lluvias de mayo a noviembre y un periodo seco de diciembre a abril. La precipitación, promedia anual es de $1.380 \mathrm{~mm}$, con una temperatura media anual de $28^{\circ} \mathrm{C}$ y humedad relativa de $81 \%$ (Estación climatológica CORPOICA-Turipana\#). Según la clasificación de Holdrige (14) la zona se clasifica ecológicamente como bosque seco tropical (bs-T).

Los sistemas silvopastoriles en los cuales se realizó el experimento fueron establecidos en el año 1998, con diferencia en su estructura y complejidad vegetal (15). La Tabla 1 resume la densidad de las especies arbustivas en cada uno de los arreglos establecidos en las cuales se consideró como especies de ramoneo a Leucaena (Leucaena leucocephala) y Totumo (Crecentia cujete), como especies arbóreas Guacimo (Guazuma ulmifolia), Campano (Albizia saman) y Cañafistula (Cassia grandis) y como especie maderable Ceiba Roja (Pachira quinata) y Caoba (Swietenia macrophylla). El estrato herbáceo estuvo dominado en su gran mayoría (40\% en el tratamiento sin árboles y con especies arbustivas, y $60 \%$ en los tratamientos con arbóreas y maderables) por la gramínea Megathyrsus maximum y en otras proporciones por Dichanthium aristatum (34\%), Cynodon nlemfuensis (13\%), otras especies de gramíneas (5\%) y leguminosas herbáceas (8\%)

Tabla 1. Densidades (plantas ha ${ }^{-1}$ ) de los diferentes estratos en cada arreglo silvopastoril evaluado.

\begin{tabular}{|c|c|c|c|c|c|c|}
\hline \multirow{2}{*}{ Estrato } & \multirow{2}{*}{ Especie } & \multicolumn{5}{|c|}{ Arreglos silvopastoriles } \\
\hline & & Pasto & Pasto-Arbust & Pasto-Arbor & Pasto-Arbust-Arbor & Pasto-Arbust-Arbor-Mad \\
\hline \multirow{2}{*}{ Arbustos } & $\begin{array}{l}\text { Totumo Crecentia } \\
\text { cujete }\end{array}$ & - & 209 & - & 161 & 161 \\
\hline & $\begin{array}{l}\text { Leucaena Leucaena } \\
\text { leucocephala }\end{array}$ & - & 208 & - & - & - \\
\hline \multirow{3}{*}{$\begin{array}{l}\text { Arboles } \\
\text { frutos }\end{array}$} & $\begin{array}{l}\text { Guácimo Guazuma } \\
\text { ulmifolia }\end{array}$ & - & - & 12 & 12 & 12 \\
\hline & $\begin{array}{l}\text { Caña Fístula Cassia } \\
\text { grandis }\end{array}$ & - & - & 12 & 12 & 12 \\
\hline & $\begin{array}{l}\text { Samán Albizia } \\
\text { saman }\end{array}$ & - & - & 12 & 12 & 12 \\
\hline \multirow{2}{*}{$\begin{array}{l}\text { Arboles } \\
\text { Maderables }\end{array}$} & $\begin{array}{l}\text { Caoba Swietenia } \\
\text { macrophylla }\end{array}$ & - & - & - & - & 12 \\
\hline & $\begin{array}{l}\text { Ceiba Pachira } \\
\text { quinata }\end{array}$ & - & - & - & - & 12 \\
\hline
\end{tabular}


El área experimental evaluada contó con una extensión de 30 ha, en las cuales se consideró un diseño de bloques completos al azar para controlar el efecto de drenaje en el lote, estableciendo tres bloques con una condición de drenaje deficiente, moderado y óptimo (15). Cada tratamiento estuvo constituido por 2 hectáreas y para efectos de manejo de pastoreo fue dividido en franjas de $4000 \mathrm{~m}^{2}$ cada una.

Características de los animales y manejo de pastoreo: Se utilizaron 20 animales provenientes de un cruce Romosinuano (50\%), Holstein (25\%) y Cebú comercial (25\%) con un peso promedio de 436 $\pm 64,2 \mathrm{~kg}$, de dos y tres partos, y condición corporal entre 3,5 y 4 puntos de acuerdo con Hess et al (16). La producción de leche de estas vacas varió entre 2,6 y 3,4l/vaca/día, con 56 28 días en lactancia y edad promedio de 52 $\pm 3,9$ meses. A cada tratamiento se le asignó al azar 5 vacas, las cuales fueron manejadas bajo un esquema de pastoreo rotacional de 2 días de ocupación y 28 días de descanso ( 15 franjas). En cada uno de los tratamientos se ofertó sal mineralizada al $8 \%$ de fósforo y agua a voluntad.

Colecta de muestras y análisis de laboratorio: Las muestras de heces fueron colectadas vía rectal en cada uno de los tratamientos evaluados. Para tal fin, fueron construidos corrales de manejo en cada tratamiento con el objeto de obtener las heces en el mismo sitio experimental.

Cada muestra fue obtenida en dos rotaciones consecutivas, considerando que los animales estuvieran al final de la rotación de cada tratamiento. En cada ocasión se colectó vía rectal aproximadamente 800 gramos de heces frescas en las horas de la mañana (6:00) y de la tarde (17:00 h); las cuales fueron almacenadas y rotulada en bolsas de cierre hermético. Para proceder al análisis, las muestras de la mañana y de la tarde obtenidas de un mismo animal fueron homogenizadas para obtener una muestra representativa.

Después de tomadas, las muestras fueron enviadas directamente al Laboratorio de Nutrición Animal del C.I. Turipaná, donde se procedió al secado en estufa de ventilación forzada a $60^{\circ} \mathrm{C}$ por 48 horas, para posteriormente ser molidas en malla de $1 \mathrm{~mm}$ en un molino tipo estacionario. En cada una de las muestras se determinó el contenido de nitrógeno por el método de Kjeldalh y la materia orgánica por combustión completa (17) y el FDN según el método descrito por Van Soest et al (18). De igual forma se determinó el contenido de nitrógeno en el residuo del FDN.

Determinación de la calidad de la dieta: La digestibilidad de la dieta consumida se estimó aplicando la ecuación de Lukas et al (19), a partir de la proteína cruda fecal, la cual se obtuvo por multiplicación del nitrógeno fecal por el factor 6.25 de acuerdo a lo descrito por Dintzis et al (20). De igual forma, el contenido de proteína cruda consumida se estimó a partir de la proteína cruda fecal, siguiendo las ecuaciones descritas por Hakkina et al (21), Holecheck et al (22) y Mcollum (23) (Tabla 2). 
Tabla 2. Ecuaciones de regresión entre el contenido de proteína fecal y el contenido de proteína cruda en bovinos.

\begin{tabular}{|l|c|c|c|c|}
\hline \multirow{2}{*}{ Autores } & \multicolumn{4}{c|}{ Modelos lineales, de la forma } \\
\cline { 2 - 5 } & $\mathbf{a}$ & $\mathbf{b}$ & $\mathbf{r}$ & $\mathbf{n}$ \\
\hline Holecheck 1982 & -0.27 & 0.84 & 0.83 & 48 \\
\hline $\begin{array}{l}\text { Hinnant vacas 1979 tomado de } \\
\text { Holecheck 1982 }\end{array}$ & 0.11 & 0.79 & 0.88 & 4 \\
\hline $\begin{array}{l}\text { Hinnant novillos 1979 tomado de } \\
\text { Holecheck 1982 }\end{array}$ & 0.09 & 0.66 & 0.9 & 4 \\
\hline $\begin{array}{l}\text { Hakkila 1988 } \\
\text { Wofford et al. 1985 tomado de } \\
\text { Hakkila 1988 }\end{array}$ & -0.11 & 1.3 & 0.67 & 6 \\
\hline $\begin{array}{l}\text { Squires y Shiebert 1983 tomado de } \\
\text { Hakkila 1988 }\end{array}$ & 0.05 & 0.94 & 0.38 & 12 \\
\hline $\begin{array}{l}\text { Cordova 1977 tomado de Hakkila } \\
\text { 1988 }\end{array}$ & -1.06 & 1.53 & 0.69 & 14 \\
\hline $\begin{array}{l}\text { Bredon et al. 1963 tomado de } \\
\text { Hakkila 1988 }\end{array}$ & -1.04 & 1.62 & 0.92 & 14 \\
\hline $\begin{array}{l}\text { Arthun 1981 tomado de Hakkila } \\
\text { 1988 }\end{array}$ & -1.35 & 1.78 & 0.62 & 4 \\
\hline $\begin{array}{l}\text { Arman 1978 tomado de Hakkila } \\
\text { 1988 Bos taurus }\end{array}$ & -2.54 & 1.87 & 0.9 & 10 \\
\hline Mcollum 1990 & -0.17 & 0.79 & 0.74 & 20 \\
\hline
\end{tabular}

Análisis estadístico: Los datos fueron colectados y almacenados en una hoja de cálculo para su posterior análisis. Se empleó un diseño de bloques completos al azar con tres repeticiones (drenaje deficiente, moderado y óptimo) y 5 tratamientos (Pasto, Pasto-Arbust, Pasto-Arbór, Pasto-Arbust-Arbór y Pasto-Arbust-ArbórMad). Para el análisis de la información se empleó el procedimiento MIXED del paquete estadístico SAS (24), teniendo como efectos fijos los tratamientos y como efecto aleatorio el efecto del bloqueo según el siguiente modelo:

$\mathrm{y}_{\mathrm{ij}}=\mu+\mathrm{T}_{\mathrm{i}}+\mathrm{B}_{\mathrm{j}}+\varepsilon_{\mathrm{ij}}$

Donde:

$\mathrm{y}_{\mathrm{ij}}=$ Variable respuesta en el $\mathrm{i}$-esimo tratamiento y $\mathrm{j}$-esimo bloque.

$\mu=$ Media general

$\mathrm{T}_{\mathrm{i}}=$ efecto del i-esimo tratamiento(Pasto,Pasto-Arbust,Pasto-Arbor,Pasto-Arbust-Arbor y Pasto-ArbustArbor-Mad)

$B_{j}=$ Efecto del $j$-esimo bloque $(1,2$ y 3$) \mathrm{N}(0,1) \sigma_{B}^{2}$

$\varepsilon_{\mathrm{ij}}=$ Error idd $\mathrm{N}(0,1) \sigma^{2}$

En caso de rechazo la hipótesis de igualdad de tratamientos, se empleó la prueba de separación de medias de Tukey, con un alfa de 0.05. Para el caso de la proteína cruda en la dieta, se corrieron todos los modelos propuestos en la Tabla 1 con base en el nitrógeno fecal estimado para cada tratamiento y se seleccionó el modelo más parsimonioso con base en los estadísticos AIC y BIC.

\section{Resultados}

La Tabla 3 presenta los resultados para la composición fecal de las muestras obtenidas en cada uno de los arreglos silvopastoriles evaluados. Solo se reportaron diferencias significativas para la variable PC. Los tratamientos Pasto, Pasto-Arbust y Pasto-Arbor presentaron respectivamente 13.28, 15.97 y 12.42 g PC $\mathrm{MO}^{-1}$ menos que tratamientos que incluye especies maderables. 
Tabla 3. Medias de mínimos cuadrados de la composición fecal bajo los modelos silvopastoriles estudiados.

\begin{tabular}{|c|c|c|c|c|c|c|}
\hline \multirow[b]{2}{*}{ Tratamiento } & MO & FDN & PC & NIDN & NSDN & RNDN \\
\hline & $\% \mathrm{MS}$ & $\% \mathrm{MS}$ & $\begin{array}{l}\mathrm{g} \bullet \mathrm{kg} \\
\mathrm{MO}^{-1}\end{array}$ & $\begin{array}{l}\mathrm{g} \bullet \mathrm{kg} \\
\mathrm{MO}^{-1}\end{array}$ & $\begin{array}{l}\mathrm{g} \bullet \mathrm{kg} \\
\mathrm{MO}^{-1}\end{array}$ & $g \bullet g^{-1}$ \\
\hline Pasto & 76.90 & 64.51 & $119,77^{\mathrm{b}}$ & 9.63 & 9.53 & 1.02 \\
\hline Pasto-Arbust & 78.74 & 63.81 & $116,62^{b}$ & 8.93 & 9.73 & 1.22 \\
\hline Pasto-Arbor & 79.22 & 62.48 & $118,91^{\mathrm{b}}$ & 8.22 & 10.81 & 1.48 \\
\hline Pasto-Arbust-Arbor & 77.11 & 62.11 & $\begin{array}{c}124,15 \\
a b\end{array}$ & 8.21 & 11.65 & 1.62 \\
\hline $\begin{array}{l}\text { Pasto-Arbust-Arbor- } \\
\text { Mad }\end{array}$ & 77.50 & 62.28 & $132,19^{a}$ & 9.68 & 11.47 & 1.29 \\
\hline \begin{tabular}{|l|} 
Significancia \\
Estadística
\end{tabular} & $P>0.05$ & $P>0.05$ & $P<0,01$ & $P>0.05$ & $P>0.05$ & $P>0.05$ \\
\hline \multicolumn{7}{|c|}{$\begin{array}{l}\text { MO: Materia Orgánica; FDN: Fibra en Detergente Neutro; PC: Proteína } \\
\text { Cruda; NIDN: Nitrógeno Insoluble en Detergente Neutro; NSDN: Nitrógeno } \\
\text { Soluble en Detergente Neutro: RNDN: relación nitrógeno soluble a nitrógeno } \\
\text { insoluble fecal Letras diferentes entre promedios en la misma columna } \\
\text { indican diferencia estadistica significativa } p<0.05 \text {. }\end{array}$} \\
\hline
\end{tabular}

Los resultados obtenidos de la estimación de la digestibilidad y el contenido de proteína cruda en la dieta seleccionada por los animales en los diferentes arreglos evaluados se presentan en la Tabla 4. Dado que el modelo seleccionado para estimar la digestibilidad y la proteína cruda en la dieta consumida por los animales en cada uno de los sistemas silvopastoriles evaluados actúa como una constante, los resultados reflejan el mismo comportamiento del nitrógeno fecal, observado en la Tabla 3.

Los resultados obtenidos reflejaron que en los sistemas silvopastoriles la digestibilidad de la dieta consumida varió $-1.1,-0.11,2.0$ y 5.01\%, y la proteína cruda consumida 2.8, -0.3, 5.4 y 14.3\% para los tratamientos Pasto-Arbust, Pasto-Arbor, Pasto-Arbust-Arbor y Pasto-Arbust-Arbor-Mad respectivamente, con relación a los datos estimados en el tratamiento sin árboles.

Tabla 4. Estimación de la digestibilidad y contenido de proteína cruda de la dieta seleccionada por los animales en los tratamientos, usando el contenido de nitrógeno fecal como estimador

\begin{tabular}{|l|c|c|c|c|c|}
\hline & Pasto & Pasto-Arbust & Pasto-Arbor & Pasto-Arbust-Arbor & Pasto-Arbust-Arbor-Mad \\
\hline $\begin{array}{l}\text { Digestibilidad } \\
\text { de la materia } \\
\text { orgánica (\%) }\end{array}$ & 62.13 & 61.45 & 62.06 & 63.37 & 65.24 \\
\hline $\begin{array}{l}\text { Proteina Cruda } \\
\text { consumida } \\
\text { (aMO) }\end{array}$ & 10.57 & 10.28 & 10.54 & 11.14 & 12.08 \\
\hline
\end{tabular}

\section{DISCUSIÓN}

El nitrógeno fecal está compuesto por dos fracciones, una asociada al nitrógeno dietario no absorbido o indigerible de origen vegetal y otra asociada al nitrógeno metabólico, el cual se considera que no proviene de la dieta y que a se compone a su vez de dos fuentes, una microbial asociada con las membranas y/o contenidos celulares indigeribles de las bacterias ruminales junto con las bacterias producto de la fermentación en el tracto posterior y otra endógena, la cual proviene de varias fuentes como células epiteliales, mucus y residuos de los jugos digestivos y biliares $(7,11,18)$. El material endógeno en las heces es fermentado en una cantidad 
tal que la separación de los componentes endógeno y microbial no tiene significado biológico ( 10 al $15 \%$ de la fracción metabólica) (18). Por otra parte, es considerado que casi todo el nitrógeno en las heces es de origen microbial, siendo muy poco el proveniente del alimento, por tal motivo, el NF puede proveer estimaciones indirectas del material microbial en heces $(18,25)$.

En la caracterización química del nitrógeno fecal se considera que no hay proteína de la dieta potencialmente digestible y que el material metabólico es soluble en detergente neutro (NSDN) $(10,26)$, salvo la queratina presente en las células del tracto gastrointestinal (18), mientras que el nitrógeno insoluble en detergente neutro (NIDN) contiene el componente dietario no digerible que puede estar asociado a la fibra, lignina y/o a taninos (25). Por tanto, la concentración de la fracción de nitrógeno en las heces que aumenta por la pérdida de material fermentable desde el rumen al tracto posterior y que refleja la fracción metabólica (principalmente membranas y/o contenidos celulares indigeribles de bacterias ruminales) se puede medir directamente restando el contenido de nitrógeno en el residuo en detergente neutro o ácido, que corresponde al nitrógeno fecal insoluble, del contenido de nitrógeno total en la materia fecal (18).

De lo anterior se puede inferir que el nitrógeno fecal es en gran parte material microbial indigerible. Por tanto, cualquier variable asociada al animal o a la dieta que influya sobre el crecimiento y/o eficiencia microbial tendrá un efecto sobre la concentración metabólica fecal de origen bacteriano y por tanto sobre la concentración total de nitrógeno fecal (27).

En el presente estudio se observa como a medida que avanza la complejidad estructural de los sistemas silvopastoriles (inclusión de especies arbustivas, arbóreas y maderables), incrementa la digestibilidad y la proteína cruda en la dieta consumida estimadas a partir del nitrógeno fecal. Diferentes autores han concluido que la inclusión de especies arbustivas en la dieta de los rumiantes contribuye a mejorar el aprovechamiento de la gramínea base, debido al incremento del contenido de proteína y de la digestibilidad en la dieta, probablemente asociado a su bajo contenido de FDN en las especies arbustivas $(28,29,30)$.

Molina et al (31) utilizando la técnica del politúnel informan que la inclusión de un $27 \%$ Leucaena leucocephala en la dieta de terneras Lucerna que consumen Megathyrsys maximum y Cynodon plectostachus, mejoró la digestibilidad de la dieta en un $5.8 \%$, comparada con una dieta tradicional de solo gramíneas (54\% M. maximum y 46\% C. plectostachus). Contrario a esto, Cuartas et al (32) empleando la técnica de n-alcanos, no reportó diferencia significativa entre la digestibilidad (53.4 SSPi vs 53.8 Monocultivo) de la dieta consumida por novillos de cruces no definidos ( $248 \pm 23.1 \mathrm{~kg}$ ), probablemente atribuido a la gran variabilidad que registra esta técnica de determinación de consumo in vivo.

Con relación al aumento en el contenido de proteína cruda consumida en la dieta, se ha reportado que el efecto de la complejidad vegetal y específicamente, la presencia de árboles y arbustos leguminosos, contribuyen a aumentar la concentración de proteína cruda en las especies herbáceas asociadas al consumo de rumiantes en sistemas silvopastoriles. Al respecto, Barragán et al (33), evaluando los sistemas silvopastoriles descritos en este trabajo, identificó un efecto significativo de la complejidad del arreglo silvopastoril y el contenido de proteína cruda en la gramínea M. maximum, al pasar de $9.7 \%$ de PC en el tratamiento sin árboles, a $12.6 \%$ PC en el tratamiento SSP que incluye especies arbustivas, arbóreas y maderables. Diferentes autores han reportado cambios en la PC de la gramínea M. maximum bajo condiciones de sombrío de árboles (34-35), estos incrementos de proteína han sido asociados a los efectos de reciclaje de nutrientes (caída de hojarasca) que se evidencia bajo ambientes silvopastoriles $(36,37)$ y al desarrollo de gramíneas bajo la cobertura de árboles leguminosos (38,39).

\section{Conclusiones}

La estimación indirecta de la digestibilidad y de la proteína cruda consumida en la dieta a través del nitrógeno fecal, ambos elementos como indicadores integrales de la calidad de la dieta que pudo ser consumida reflejaron los efectos diferenciales de la complejidad vegetal en cada uno de los arreglos silvopastoriles evaluados, la cual 
puede estar influenciando la capacidad de consumo, selectividad de la dieta por parte de los animales y las interacciones biológicas que se generan en cada uno de los sistemas silvopastoriles y que inducen a una mayor proporción de proteína cruda en las especies que componen la estructura vegetal de cada arreglo.

\section{REFERENCIAS}

1. Braghieri A, Pacelli C, De Rosa G, Girolami A, De Palo P, Napolitano F. Podolian beef production on pasture and in confinement. Animal. 2011; 5(6):927-937. DOI: https://doi.org/10.1017/S1751731110002685 PMid:22440032

2. Coppa M, Farruggia A, Pradel P, Lombardi G, Martin B. An improved grazed class method to estimate species selection and dry matter intake by cows at pasture. Ital J Anim Sci. 2011; 10(1):58-65. DOI: https:// doi.org/10.4081/ijas.2011.e13

3. Wang L, Wang D, Bai Y, Jiang G, Liu J, Huang Y, et al. Spatial distributions of multiple plant species affect herbivore foraging selectivity. Oikos. 2010; 119(2):401-408. DOI: https://doi.org/10.1111/j.1600-0706.2009.17774.x

4. Ramirez-Lozano RG, Gonzalez-Rodriguez H, Ledezma-Torres R. Nutritional evaluation of Senegalia greggii and Prosopis juliflora as browse supplements for sheep. Indian Journal Of Animal Research. 2018; 52(9):1304-1308.

5. Stejskalová M, Hejcmanová P, Pavlů V, Hejcman M. Grazing behavior and performance of beef cattle as a function of sward structure and herbage quality under rotational and continuous stocking on species-rich upland pasture. Anim Sci J. 2013; 84(8):622-629. DOI: https://doi.org/10.1111/asj.12048 PMid:23607767

6. Agreil C, Meuret M. An improved method for quantifying intake rate and ingestive behaviour of ruminants in diverse and variable habitats using direct observation. Small Rumin Res. 2004; 54(1-2):99-113. DOI: https:// doi.org/10.1016/j.smallrumres.2003.10.013

7. Schlecht E, Susenbeth A. Estimating the digestibility of Sahelian roughages from faecal crude protein concentration of cattle and small ruminants. J Anim Physiol Anim Nutr (Berl). 2006; 90(9-10):369-379. DOI: https:// doi.org/10.1111/j.1439-0396.2005.00596.x PMid:16958793

8. Kozloski G V., Oliveira L, Poli CHEC, Azevedo EB, David DB, Ribeiro Filho HMN, et al. Faecal nitrogen excretion as an approach to estimate forage intake of wethers. J Anim Physiol Anim Nutr (Berl). 2014; 98(4):659-666. DOI: https://doi.org/10.1111/jpn.12118 PMid:23931613

9. Wehausen JD. Fecal Measures of Diet Quality in Wild and Domestic Ruminants. JSTOR. 1995; 59(4):816-823.

10. Leslie DM, Bowyer RT, Jenks JA. Facts From Feces: Nitrogen Still Measures Up as a Nutritional Index for Mammalian Herbivores. J Wildl Manage [Internet]. 2008; 72(6):1420-1433. DOI: https:// doi.org/10.2193/2007-404

11. Peripolli V, Prates ÊR, Barcellos JOJ, Neto JB. Fecal nitrogen to estimate intake and digestibility in grazing ruminants. Anim Feed Sci Technol [Internet]. 2011; 163(2-4):170-176. DOI: https://doi.org/10.1016/ j.anifeedsci.2010.11.008

12. Wang CJ, Tas BM, Glindemann T, Rave G, Schmidt L, Weißbach F, et al. Fecal crude protein content as an estimate for the digestibility of forage in grazing sheep. Anim Feed Sci Technol. 2009; 149(3-4):199-208. DOI: https://doi.org/10.1016/j.anifeedsci.2008.06.005

13. Waldrip HM, Todd RW, Cole NA. Prediction of nitrogen excretion by beef cattle: A meta-analysis. J Anim Sci. 2013; 91(9):4290-4302. DOI: https://doi.org/10.2527/jas.2012-5818 PMid:23825341

14. Holdridge LR (Leslie R. Forest environments in tropical life zones; a pilot study. 1st ed.]. Oxford,: Pergamon Press; 1971 [cited 2018 May 3]. https://searchworks.stanford.edu/view/609670

15. Cajas-Giron YS, Sinclair FL. Characterization of multistrata silvopastoral systems on seasonally dry pastures in the Caribbean Region of Colombia. Agrofor Syst. 2001; 53(2):215-225. DOI: https://doi.org/10.1023/ A: 1013384706085

16. Hess HD, Díaz T, Florez hernando. Guia Para la Evaluacion de la Condicion Corporal de Vacas en Sistemas Doble Proposito. Bogotá: Corpoica; 1999. 
17. AOAC. Official methods of Analysis. 18th edition. Associatio. Arlington, VA.; 2005.

18. Van Soest PJ, Robertson JB, Lewis BA. Methods for Dietary Fiber, Neutral Detergent Fiber, and Nonstarch Polysaccharides in Relation to Animal Nutrition. J Dairy Sci. 1991; 74(10):3583-97. DOI: https:// doi.org/10.3168/jds.S0022-0302(91)78551-2

19. Lukas M, Sudekum KH, Rave G, Friedel K, Susenbeth A. Relationship between fecal crude protein concentration and diet organic matter digestibility in cattle. J Anim Sci. 2005; 83:1332-1344. DOI: https:// doi.org/10.2527/2005.8361332x PMid:15890810

20. Dintzis FR, Cavins JF, Graf E, Stahly T. Nitrogen-to-Protein Conversion Factors in Animal Feed and Fecal Samples. J Anim Sci. 1988; 66(1):5-11. DOI: https://doi.org/10.2527/jas1988.6615 PMid:3366716

21. Hakkila MD, Holechek JL, Wallace JD, Anderson DM, Cardenas M. Diet and forage intake of cattle on desert grassland range. J Range Manag. 1987; 40:339-42. DOI: https://doi.org/10.2307/3898733

22. Holechek JL, Vavra M, Arthun D. Relationships between Performance, Intake, Diet Nutritive Quality and Fecal Nutritive Quality of Cattle on Mountain Range. Journal of Range Management. 1982; 35(6):741-744. DOI: https://doi.org/10.2307/3898253

23. McCollum F. Relationships among fecal nitrogen, diet nitro- gen, and daily gain of steers grazing tallgrass prairie. Okla Agric Exp Stn. 1990; MP-129:232-5.

24. SAS Institute Inc. Introducción a la programación en SAS • [Internet]. Cary, NC, USA; 2014. https:// support.sas.com/documentation/cdl_alternate/es/webeditorgs/67431/PDF/default/webeditorgs.pdf

25. Schwarm A, Schweigert M, Ortmann S, Hummel J, Janssens GPJ, Streich WJ, et al. No easy solution for the fractionation of faecal nitrogen in captive wild herbivores: Results of a pilot study. J Anim Physiol Anim Nutr (Berl). 2009; 93(5):596-605. DOI: https://doi.org/10.1111/j.1439-0396.2008.00842.x PMid:19178609

26. Powell JM, Broderick GA, Grabber JH, Hymes-Fecht UC. Technical note: Effects of forage protein-binding polyphenols on chemistry of dairy excreta. J Dairy Sci. 2009; 92(4):1765-1769. DOI: https://doi.org/10.3168/ jds.2008-1738 PMid:19307659

27. Dong RL, Zhao GY, Chai LL, Beauchemin KA. Prediction of urinary and fecal nitrogen excretion by beef cattle. J Anim Sci. 2014; 92(10):4669-4681. DOI: https://doi.org/10.2527/jas.2014-8000 PMid:25149338

28. Gaviria-Uribe X, Naranjo-Ramírez JF, Bolívar-Vergara DM, Barahona-Rosales R. Consumo y digestibilidad en novillos cebuínos en un sistema silvopastoril intensivo. Arch Zootec. 2015; 64(245):21-27. DOI: https:// doi.org/10.21071/az.v64i245.370

29. Murgueitio E, Chará J, Barahona R, Cuartas C, Naranjo J. Los Sistemas Silvopastoriles Intensivos (Sspi), Herramienta De Mitigación y Adaptación Al Cambio Climático. Trop Subtrop Agroecosystems. 2014; 17(3):501-507.

30. Agudelo Carmona JC. Efecto de la utilización de arbóreas y arbustivas forrajeras sobre la dinámica digestiva en bovinos. Rev Lasallista Investigación. 2007; 4(1):40-50.

31. Molina IC, Donney`s G, Montoya S, Rivera JE, Villegas G, Chará J, et al. La inclusión de Leucaena leucocephala reduce la producción de metano de terneras lucerna alimentadas con Cynodon plectostachyus y Megathyrsus maximus. Livest Res Rural Dev. 2015; 27(5). https://www.lrrd.org/lrrd27/5/moli2705cit.htm

32. Cuartas CA, Naranjo JF, Tarazona A, Correa G, Barahona R. Dry Matter And Nutrient Intake And Diet Composition In Leucaena leucocephala - Based Intensive Silvopastoral Systems. Trop Subtrop Agroecosystems. 2015; 18:303-11.

33. Barragán Hernández W, Mahecha-Ledesma L, Cajas-Girón Y. Efecto de sistemas silvopastoriles en la producción y composición de la leche bajo condiciones del valle medio del río Sinú, Colombia. Rev Colomb Cienc Anim. 2016; 8(2):187-196. DOI: https://doi.org/10.24188/recia.v8.n2.2016.186

34. Wilson JR, Wild DMW. Improvement of nitrogen nutrition and grass growth under shading. In: Shelton HM, Stur WW. (Editors) Forages Plant Crops. Goanna Print Ply Ltd: Canberra, Australia; 1991.

35. Medinilla-Salinas L, Vargas-Mendoza MD la C, López-Ortiz S, Ávila-Reséndiz C, Campbell WB, GutiérrezCastorena $\mathrm{M}$ del C. Growth, productivity and quality of Megathyrsus maximus under cover from Gliricidia sepium. Agrofor Syst. 2013; 87(4):891-9. DOI: https://doi.org/10.1007/s10457-013-9605-1 
36. Martínez J, Cajas YS, León JD, Osorio NW. Silvopastoral systems enhance soil quality in grasslands of Colombia. Appl Environ Soil Sci. 2014. DOI: https://doi.org/10.1155/2014/359736

37. Avendaño-Yánez M de la L, López-Ortiz S, Perroni Y, Pérez-Elizalde S. Leguminous trees from tropical dry forest generate fertility islands in pastures. Arid L Res Manag. 2018; 32(1):57-70. DOI: https:// doi.org/10.1080/15324982.2017.1377782

38. Pentón G, Blanco F. Influencia de la sombra de los arboles en la composicion quimica y el rendimiento de los pastos. Pastos Y Forrajes. 1997; 20(2):101-10. https://payfo.ihatuey.cu/index.php? journal $=$ pasto\&page $=$ article\&op $=$ view \&path $\% 5 \mathrm{~B} \% 5 \mathrm{D}=1301 \&$ path $\% 5 \mathrm{~B} \% 5 \mathrm{D}=803$

39. Treydte AC, Heitkönig IMA, Prins HHT, Ludwig F. Trees improve grass quality for herbivores in African savannas. Perspect Plant Ecol Evol Syst. 2007; 8(4):197-205. DOI: https://doi.org/10.1016/ j.ppees.2007.03.001

Los autores permiten a RECIA reimprimir el material publicado en él. En caso de que un autor quiera traducir o usar una publicación parcial o completa de nuestro Diario, el autor debe obtener un permiso por escrito del editor de la revista.

CC BY

\section{INFORMACIÓN ADICIONAL}

Descargo de responsabilidades: La presente investigación se llevó a cabo en el marco del proyecto "Valoración Integral de los Beneficios de Sistemas Silvopastoriles de Estratos Múltiples sobre la Sostenibilidad del Suelo y la Productividad Animal en el Sistema Doble Propósito de la Región Caribe de Colombia" financiado por el Ministerio de Agricultura y Desarrollo Rural en convocatoria 2010, y ejecutado por CORPOICA. 